\title{
REVIEW
}

\section{Biased agonism: a novel paradigm in G protein-coupled receptor signaling observed in acquired hypocalciuric hypercalcemia}

\author{
Noriko Makita and Taroh Iiri \\ Department of Endocrinology and Nephrology, The University of Tokyo School of Medicine, Tokyo 113-8655 Japan
}

\begin{abstract}
The classical model of G protein-coupled receptor (GPCR) activation is the two-state model, in which the GPCR exists in equilibrium between an active and inactive state. Based on this model, GPCR ligands have been classified as agonists, inverse agonists, or antagonists depending on their actions in shifting this equilibrium. Recently, however, accumulating evidence has indicated that GPCRs may exist in multiple active and inactive conformational states. In this situation, each ligand recognizes and stabilizes a specific conformation of the GPCR, leading to a set of specific biological effects. Based on this new model, a unique agonist or a combination of the usual agonist and an allosteric modulator may enable activation of a specific signaling pathway via a GPCR that activates multiple signals (biased agonism, functional selectivity). The calcium-sensing receptor autoantibody that we have identified in the serum of a patient with acquired hypocalciuric hypercalcemia (AHH) is the first example of a biased allosteric modulator of a GPCR working in a pathophysiological context. Our findings may indicate the presence of physiological allosteric modulators and provide new directions for the future drug development.
\end{abstract}

Key words: G protein-coupled receptor (GPCR), Biased agonism, Calcium-sensing receptor, Acquired hypocalciuric hypercalcemia $(\mathrm{AHH})$, Protean agonism

G PROTEIN-COUPLED RECEPTORS (GPCRs) process a variety of extracellular signals related to cognition, sense, hormonal and metabolic control, cardiovascular regulation, and the immune system, and relay this information to intracellular signaling pathways [1-3]. This signaling system principally consists of GPCRs, G proteins, and effectors. GPCRs are encoded by about 1,000 genes and represent one of the largest families in the human genome. The $\mathrm{G}$ protein consists of $G \alpha, G \beta$, and $G \gamma$ subunits encoded separate genes from a pool of at least $16 \mathrm{G} \alpha, 6 \mathrm{G} \beta$, and $12 \mathrm{G} \gamma$ genes. This pool potentially leads to about 1,000 variations of the $\mathrm{G}$ protein complex $(\mathrm{G} \alpha / \mathrm{G} \beta / \mathrm{G} \gamma)$. G proteins regulate about 100 different effectors. In contrast to this diversity, the molecular mechanism underlying the GPCR-dependent activation of $\mathrm{G}$ proteins has been evolutionarily conserved among different species. For

Submitted Oct. 29, 2013; Accepted Nov. 2, 2013 as EJ13-0453

Released online in J-STAGE as advance publication Nov. 16, 2013

Correspondence to: Noriko Makita and Taroh Iiri, Department of Endocrinology and Nephrology, The University of Tokyo School of Medicine, 7-3-1 Hongo, Bunkyo-ku, Tokyo 113-8655 Japan. E-mail: norimaki-tky@umin.ac.jp, tiiri-tky@umin.ac.jp the past 20 years, many activating or inactivating mutations of GPCRs or G proteins have been reported as the underlying causes of different diseases [4-6]. Analyses of the mutants have revealed not only the molecular pathways of many diseases but also the physiological mechanisms of GPCRs.

\section{GPCR signaling networks}

GPCR signaling networks consists of GPCRs, G proteins, and effectors. In some cases, each GPCR-G protein-effector axis works independently (Fig. 1-A). In other cases, multiple GPCRs couple to one G protein thus leading to a signaling converging (Fig. 1-B). Conversely, many examples have been reported in which one GPCR couples to multiple $\mathrm{G}$ proteins i.e. divergent signaling (Fig. 1-C). In the classical model, it has been believed that when an agonist interacts with one GPCR that couples with multiple G proteins, multiple downstream signals related to these $G$ proteins are activated to the same degree. To activate only one downstream signal in this model, an agonist needs to pair with a GPCR that specifically couples with only 
one $\mathrm{G}$ protein.

A new concept, in which a unique agonist may activate a specific signaling pathway via a GPCR that involved in multiple signaling has emerged recently (Fig. 1-D). This concept is referred to as functional selectivity or biased agonism, depending on whether the focus is the receptor or the agonist [7-11]. Moreover, an allosteric modulator, which mediates the effects of an agonist by acting on a GPCR site that differs from the agonist interaction site, may augment or attenuate signals or in unique cases may activate a specific signaling pathway [12, 13] (Fig. 1-D).

If functionally selective activation is a true physiological mechanism, numerous agonist/GPCR pairs may not be required to activate specific signaling pathways and the fine-tuning of signals may be possible dependent on the cell type or context. However, this hypothesis is not compatible with the classical model of GPCR activation.

\section{Two-state versus multi-state model}

In the classical model of GPCR activation, it has been assumed that GPCRs exist in equilibrium between active and inactive states (Fig. 2-A). This two-state model is often used to explain many of the phenomena regarding GPCR activation and inactivation. In this model, for example, the GPCRs are activated at various frequencies without agonist stimulation. This explains why these receptors show basal activity when expressed. In this classical model, it is postulated that agonists shift the GPCR equilibrium toward the active form whereas inverse agonists do the opposite, and that antagonists, especially neutral antagonists, inhibit agonists competitively without affecting the equilibrium.

However, several lines of evidence now lend support to an alternative multi-state model for GPCRs, in which these receptors may spontaneously adopt multiple active and inactive conformational states (Fig. 2-B). In this new multi-state model, it is hypothesized that each ligand recognizes and stabilizes a specific conformation for the GPCR, leading to a set of unique and specific biological effects. A functionally selective agonist or biased agonist is defined in this case as a unique drug that recognizes and stabilizes a "chimeric" conformation that is "on" with respect to one signaling pathway and yet "off" to another. This enables the activation of a specific signaling pathway via a GPCR that activates multiple signals.

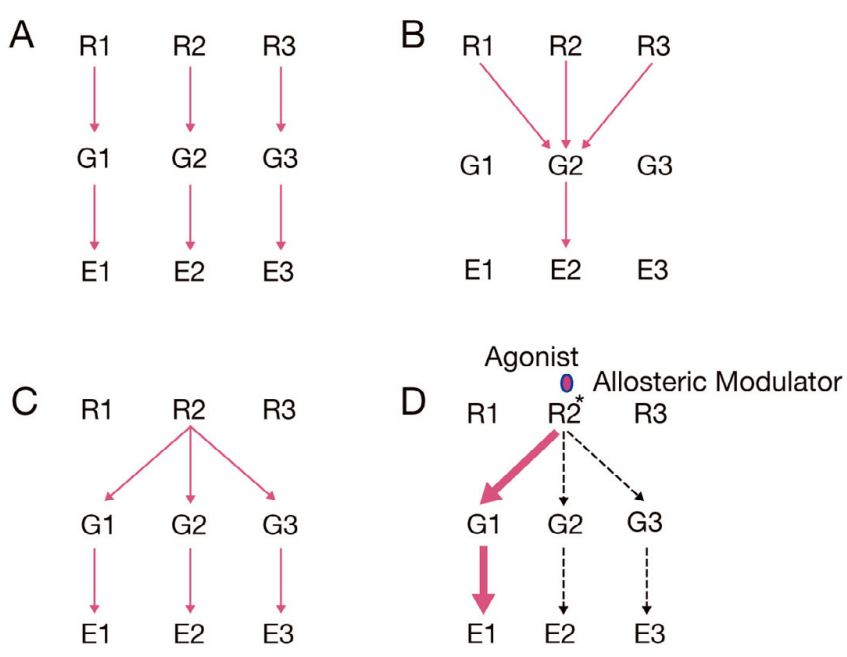

Fig. 1 Signal sorting in GPCR networks

GPCR signaling networks consist of GPCRs (R), G proteins $(G)$, and effectors (E). Signals can operate independently (A), converge (B), or diverge (C). In the classical model of GPCR, it has been believed that when an agonist activates a GPCR that couples with multiple $\mathrm{G}$ proteins, the GPCR regulates multiple downstream signals to the same degree (C). In a novel model, a unique biased agonist or a combination of usual agonist plus a biased allosteric modulator can activate a specific signaling pathway via a GPCR that activates multiple signals (D).

\section{Functional selectivity/biased agonism in GPCR pathways}

As an example that supports the multi-state model, it has been reported that in the PACAP receptor PACAP1-27 shows a higher relative efficacy for cAMP accumulation, but lower for inositol 1, 4, 5-trisphosphate (IP3) accumulation, than PACAP1-38 in LLC PK1 cells expressing PACAP receptors transiently [14]. This suggests that agonist activation of multiple signaling mechanisms is not uniform but is in fact often biased toward some but not all signaling pathways, and that agonist-selective states can produce biased agonism [15]. The findings of subsequent studies have supported this concept including ligand specific conformational changes of the $\beta 2$-adrenergic receptor $[16,17]$, different types of agonists that induce selective coupling to a distinct second messenger pathway via the Drosophila D1-like dopamine receptor expressed in Xenopus oocytes [18], and receptor ligand specific dominancy between PLC-mediated inositol phosphate (IP) accumulation and PLA2-mediated arachidonic 

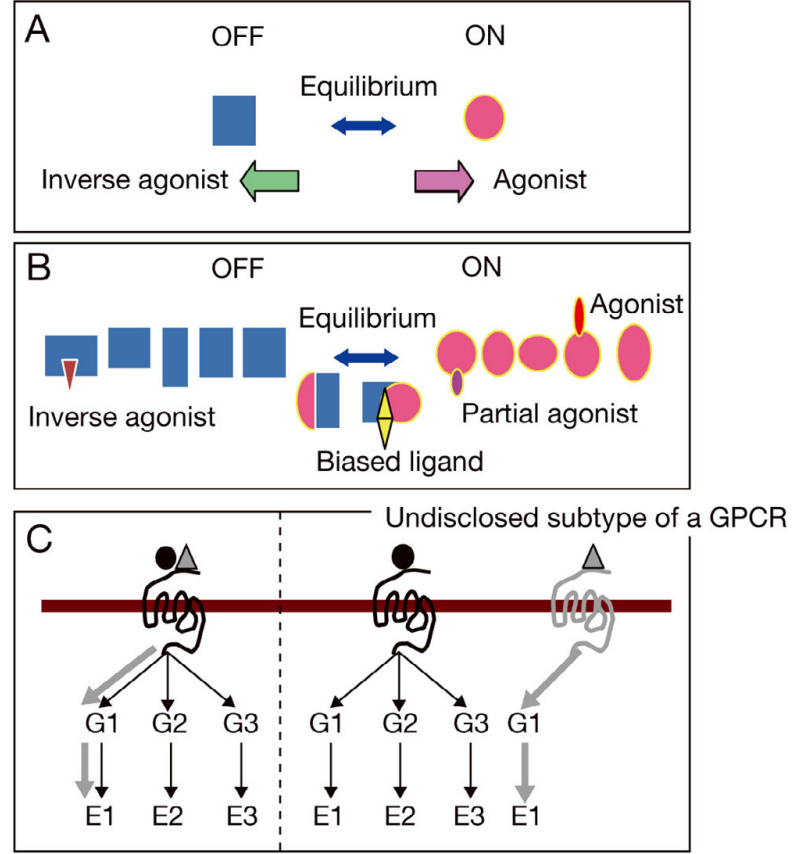

Fig. 2 Two-state model vs. multi-state model

(A) Two state model. The classical model of GPCR activation is the two-state model, in which GPCRs are assumed to exist in equilibrium between active and inactive states. It is postulated that agonists shift the equilibrium toward the active form whereas inverse agonists shift the equilibrium toward the inactive form. (B) Multi-state model. Accumulating evidence now suggests that GPCRs can spontaneously adopt multiple active and inactive conformational states. Each ligand recognizes and stabilizes a specific conformation for the GPCR, leading to a set of specific biological effects. A biased ligand (or a combination of usual agonist and a biased allosteric modulator) recognizes and stabilizes a "chimeric" conformation that is "on" to one signaling pathway but "off" to another. (C) A point of note in biased agonism. In the context of biased agonism, it is important to decide that both the normal and biased agonists act on the same single GPCR (left). An alternative possibility to be ruled out is that the two agonists work on different but closely related GPCRs (right).

acid release through human serotonin $2 \mathrm{c}(5-\mathrm{HT} 2 \mathrm{c})$ in CHO cells [19].

In the case of the PTH receptor that is known to activate Gs/adenylyl cyclase and $\mathrm{Gq} /$ phospholipase C via PTH (1-34), PTH (3-34) cannot activate adenylyl cyclase even though it retains the ability to activate ERK1/2, possibly via Gq, in rat osteoblastic cells and UMR106-01 cells [20]. In tachykinin NK2 receptors expressed in HEK293 cells, NKA increases intracellular calcium and cAMP accumulation whilst the NKA (4-10) peptide increases only intracellular calcium with- out a cAMP response, suggestive of two distinct conformational states [21]. It must be noted in this context that it is very important to determine that both the normal agonist and the biased agonist act on the same single GPCR (Fig. 2-C, left). An alternative possibility that has to be ruled out is that the two agonists work on different GPCRs, such as closely related GPCR subtypes (Fig. 2-C, right). It will be particularly important to determine if biased agonism operates via a GPCR endogenously expressed in cells.

In the history of biased agonism in GPCR pathways, a seminal study was reported by the Bouvier group in 2003 [22]. In that study, it was demonstrated that the $\beta 2$-adrenergic receptor ligands, ICI118511 and propranolol, function as inverse agonists in Gs-cAMP signaling and as partial agonists in ERK1/2 phosphorylation. The findings of this report are extremely interesting from the perspective that a unique biased ligand for one specific GPCR may work as both an agonist and an inverse agonist, depending on which signal transduction pathway is measured (Fig. 2-B).

There have been many reports showing that a GPCR activates plural $\mathrm{G}$ proteins. This seems to be at odds however with the aim of developing a specific signal in cells. In the past, it was believed that the localization of signaling machinery in cells may enable a specific signal event. As an alternative concept that may resolve this question, biased agonism may be a key mechanism. In a system expressing redundant and plural GPCRs, instead of expressing a specific GPCR/agonist pair which is time consuming and energy intensive, a specific biased agonist or biased allosteric modulator may enable a more rapid and flexible regulation of signals.

We have previously identified a unique autoantibody in a patient diagnosed with acquired hypocalciuric hypercalcemia (AHH) [23]. This autoantibody likely works as a biased allosteric modulator and stabilizes a unique conformation of the calcium sensing receptor (CaSR), which augments the Gq-PI turnover pathway and yet attenuates the Gi/o-ERK1/2 pathway. The characteristics of this autoantibody not only help to explain the mechanisms underlying AHH but also support the existence of biased agonism in GPCR signaling. At about the same time, several other examples of biased agonism in GPCR systems were reported including oxyntomodulin for the GLP-1 receptor [24], aripiprazole for the D2 receptor [25], carvedilol for the $\beta 2$ receptor [26].

As the only reported example to date of a biased 
allosteric modulator, a conformation-specific allosteric antagonist has been described for the tachykinin NK2 receptor (NK2R) [27]. LPI805 promotes destabilization of the NKA-NK2R complexes that are in a conformation that triggers cAMP production, whereas the access of NKA to the conformation that triggers intracellular calcium elevation is unchanged. In contrast, among family $\mathrm{C}$ GPCRs such as metabotropic glutamate receptors, taste receptors and the $\mathrm{GABA}_{\mathrm{B}}$ receptor, including a calcium sensing receptor, many positive and negative allosteric modulators have now been reported [28, 29]. In contrast to the autoantibodies we described in our AHH patient, however, most of these factors are not biased modulators.

\section{Calcium sensing receptor — physiology and disease}

Extracellular calcium ion controls its own concentration by stimulating the CaSR that is expressed in the parathyroid gland and renal tubular epithelium [30, 31]. When the serum calcium level increases, calciumstimulated CaSR inhibits the secretion of parathyroid hormone (PTH) through a poorly defined mechanism, in which $\mathrm{Gq} / 11$ is believed to play a key role, and regulates the serum calcium level within a normal range. In contrast, when the serum calcium level decreases, PTH secretion is stimulated, followed by normalization of serum calcium. An antagonist acting on CaSR, a loss-of-function mutation of CaSR, and a blocking antibody to CaSR mimic hypocalcemia. A heterozygous loss-of-function mutation of CaSR causes familial hypocalciuric hypercalcemia $(\mathrm{FHH})$ showing mild hypercalcemia with hypocalciuria. Brown group have reported that a blocking autoantibody to CaSR causes AHH which is a similar disease to FHH [32].

\section{Characteristics of CaSR}

CaSR belongs to the family $\mathrm{C}$ of GPCRs and contains a heptahelical domain, which plays a key role in $\mathrm{G}$ protein coupling, and a venous flytrap domain that traps the agonist. CaSR is assumed to constitutively dimerize through disulfide bonding between cystein residues 120 and 131, an event that underlies the proper functioning of this receptor. Allosteric regulation is one of the important characteristics of CaSR, similar to the taste receptors and metabotrophic glutamate receptors $[28,33]$. Allosteric modulators acting at six or seven transmembrane domains have been found to modulate the effects of calcium either positively or negatively. Positive allosteric modulators for CaSR are known as calcimimetics, and negative allosteric modulators as calcilytics. Calcimimetics in particular have been used clinically for the treatment of tertiary hyperparathyroidism in patients with end stage renal disease. Calcimimetics do not activate CaSR in the absence of calcium ions, but augment the sensitivity to these ions.

\section{An autoantibody to CaSR in an AHH patient is a biased allosteric modulator}

As described earlier, we have identified and reported a unique autoantibody in a male patient, who showed mild hypercalcemia [23]. As this patient had showed normocalcemia in the past, this condition was suspected to be acquired. In one aspect in which his hypercalcemia was accompanied by hypocalciuria, his disease was similar to FHH. We suspected that he suffered from $\mathrm{AHH}$ caused by blocking autoantibody to CaSR, which only Brown group had reported to date. In terms of immunofluorescence, his serum was found to contain autoantibodies that reacted to CaSR expressed in HEK293 cells. We next investigated how these autoantibodies modulated calcium /CaSR-dependent signals. Surprisingly, however, the autoantibody in our patient was found to augment calcium-dependent phosphatidyl inositol (PI) turnover, dependent on $\mathrm{Gq} / 11$, in contrast to blocking characteristics of the autoantibody reported by Brown and colleagues. Specifically, we found that the autoantibody from our patient did not stimulate PI turnover in the absence of calcium ions, but shifted the dose response curve of calcium-dependent IP accumulation to the left. This effect is similar to that exerted by calcimimetics working as positive allosteric modulators of CaSR. Interestingly, the autoantibody in our patient was also found to inhibit calcium-dependent ERK1/2 phosphorylation, dependent on Gi/o (at least up to the $2.5 \mathrm{mM}$ calcium level). This contrasts with the effects of calcimimetics that augment calcium-dependent ERK1/2 phosphorylation as well as calcium-dependent IP accumulation (Fig. 3A) (please also refer Fig. 4).

We thus revealed three main phenomena from our identification and characterization of the CaSR sutoantibody in our AHH patient: (1) this CaSR autoantibody works as a positive allosteric modulator in $\mathrm{Gq} / 11$ signaling, but as negative allosteric modulator in Gi/o signaling, suggesting that it functions as a biased allos- 
A

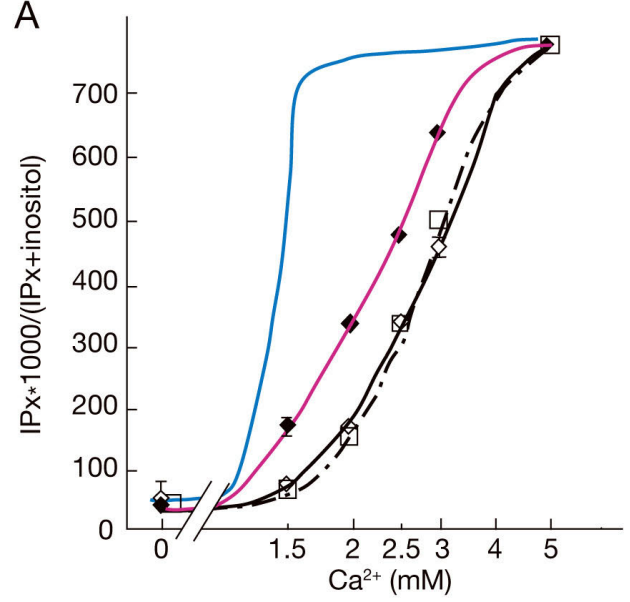

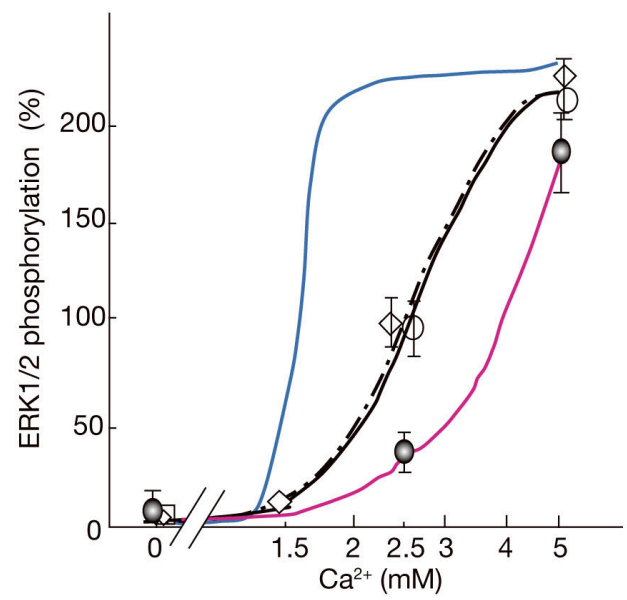

Control lgG $-\cdot-\cdot(-)$

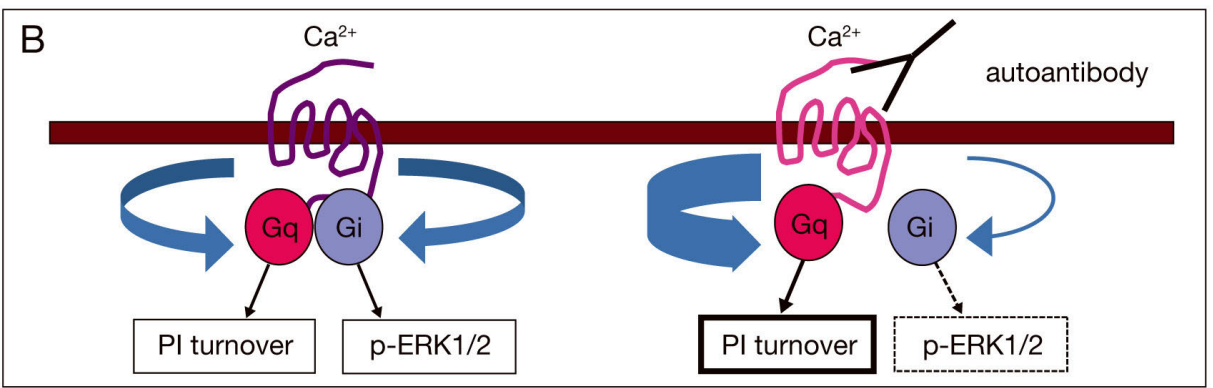

Fig. 3 Autoantibodies of our AHH patient work as biased allosteric modulators of CaSR.

(A) Gq/11 vs. Gi/o. The AHH autoantibodies work as biased allosteric modulators to CaSR, positively regulating Gq/11dependent PI turnover and yet negatively regulating Gi/o-dependent ERK1/2 phosphorylation. In contrast, a calcimimetic operates as a positive allosteric modulator to both signals. (B) Multi-states of CaSR. The data in (A) suggest that at least two different active conformations of CaSR exist. A calcium-bound form of CaSR activates both Gq/11 and Gi/o, whereas a calcium and $\mathrm{AHH}$ autoantibody-bound form of CaSR activates $\mathrm{Gq} / 11$ specifically. IPx, Inositol phosphates

teric modulator of CaSR; (2) CaSR shows at least two different active conformations, a calcium-bound form that couples with both $\mathrm{Gq} / 11$ and $\mathrm{Gi} / \mathrm{o}$, and a calcium and autoantibody-bound form that couples to $\mathrm{Gq} / 11$ specifically (Fig. 3B); (3) in clinical setting, not only $\mathrm{Gq} / 11$ but also Gi/o may play a key role in the regulation of PTH secretion.

\section{Characteristics of newly diagnosed AHH patients}

Many suspect patients of AHH have been referred to us to date, and four cases in addition to our seminal case described have been diagnosed as AHH by immunological and functional tests (unpublished, Fig. 4). Although these patients show a range of presentations from severe hypercalcemia with symptoms to mild

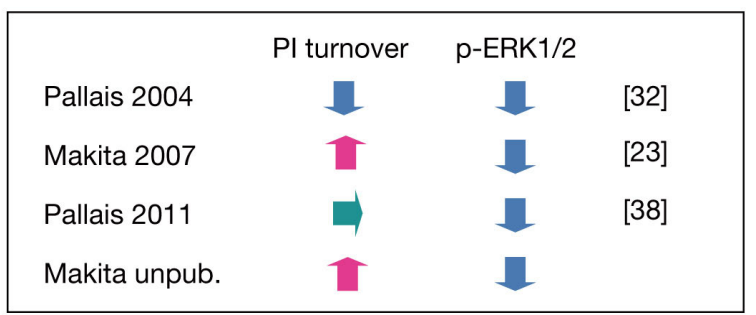

Fig. 4 The characteristics of AHH autoantibodies Brown and colleagues reported in an AHH patient blocking autoantibodies that inhibit both PI turnover and ERK phosphrylation (2004). The autoantibodies in our patient were found to operate as biased allosteric modulaotors to CaSR, augmenting Gq/11-dependent PI turnover and yet inhibiting Gi/o-dependent ERK1/2 phosphorylation (2007). Brown group subsequently reported AHH autoantibodies that inhibit ERK phosphorylation without affecting PI turnover (2011). The characteristics of the autoantibodies of new AHH patients we analyzed were similar to that of the patient we reported in 2007 (unpublished). 
hypercalcemia without symptoms, all of the autoantibodies in these cases were found to react to the extracellular domain (ECD) of CaSR, and to stimulate $\mathrm{Gq} / 11$ signals and inhibit Gi/o signals. All of our patients were elderly men with no other autoimmune disease. Moreover, in one case at least, the CaSR autoantibody was found to react to an epitope near to the flytrap domain in the ECD (unpublished data). We do not know currently whether Japanese patients are naïve to this disease or not. The key questions to be answered are in which part of the ECD is recognized by CaSR autoantibodies and how they modulate CaSR-coupling $\mathrm{G}$ proteins.

\section{Protean agonism}

A special case of GPCR-based functional selectivity is "protean" agonism, in which certain agonists may change or even reverse their effects depending on the states or systems adopted [9, 15, 34]. For example, in a quiescent system consisting mainly of receptors in an inactive state, some agonists may produce positive agonism. In contrast, in a constitutively active system involving a substantial spontaneously formed receptor active state, the same agonists may produce inverse agonism. This is because they convert the efficacious active state to a less efficacious ligand selective active state. Because the ligand effect changes in response to the system, these molecules were named protean agonists after the Greek sea-god Proteus (son of Poseidon), who could change shape at will, depending on his environment and needs [35]. As examples, the ligands for histamine $\mathrm{H} 3$ receptor (H3R) operate from full agonism to full inverse agonism, depending on the level of H3R constitutive activity [36]. Recently, we have reported that V2 receptor antagonists act as protean agonists, serving as pharmacological chaperones for inactivating V2 receptor mutants and also as inverse agonists of wild-type V2 receptor [37].

\section{Summary}

We have identified unique autoantibodies working as biased allosteric modulators of CaSR in a clinical context. Conceptually speaking, these autoantibody can switch CaSR coupling with both $\mathrm{Gq} / 11$ and Gi/o into CaSR coupling with $\mathrm{Gq} / 11$ specifically. This is therefore one clear example that the biased allosteric modulation of GPCR operates in disease. Biased agonism of GPCRs may therefore operate more generally in physiological and pathophysiological contexts. The concept of biased agonism, including protean agonism, may play a key role in developing unique new drugs as a biased agonist may switch a desirable signal "on" and an undesirable signal "off".

\section{References}

1. Gether U (2000) Uncovering molecular mechanisms involved in activation of $\mathrm{G}$ protein-coupled receptors. Endocr Rev 21: 90-113.

2. Oldham WM, Hamm HE (2008) Heterotrimeric G protein activation by G-protein-coupled receptors. Nat Rev Mol Cell Biol 9: 60-71.

3. Rosenbaum DM, Rasmussen SG, Kobilka BK (2009) The structure and function of G-protein-coupled receptors. Nature 459: 356-363.

4. Iiri T, Farfel Z, Bourne HR (1998) G-protein diseases furnish a model for the turn-on switch. Nature 394: 35-38.

5. Farfel Z, Bourne HR, Iiri T (1999) The expanding spectrum of G protein diseases. N Engl J Med 340: 10121020.

6. Lania AG, Mantovani G, Spada A (2006) Mechanisms of disease: Mutations of $\mathrm{G}$ proteins and G-proteincoupled receptors in endocrine diseases. Nat Clin Pract Endocrinol Metab 2: 681-693.
7. Christopoulos A, Kenakin T (2002) G protein-coupled receptor allosterism and complexing. Pharmacol Rev 54: 323-374.

8. Kenakin T (2005) New concepts in drug discovery: collateral efficacy and permissive antagonism. Nat Rev Drug Discov 4: 919-927.

9. Hill SJ (2006) G-protein-coupled receptors: past, present and future. Br J Pharmacol 147 Suppl 1: S27-37.

10. Urban JD, Clarke WP, von Zastrow M, Nichols DE, Kobilka B, et al. (2007) Functional selectivity and classical concepts of quantitative pharmacology. $J$ Pharmacol Exp Ther 320: 1-13.

11. Rajagopal S, Rajagopal K, Lefkowitz RJ (2010) Teaching old receptors new tricks: biasing seven-transmembrane receptors. Nat Rev Drug Discov 9: 373-386.

12. Leach K, Sexton PM, Christopoulos A (2007) Allosteric GPCR modulators: taking advantage of permissive receptor pharmacology. Trends Pharmacol Sci 28: 382389. 
13. May LT, Leach K, Sexton PM, Christopoulos A (2007) Allosteric modulation of $\mathrm{G}$ protein-coupled receptors. Annu Rev Pharmacol Toxicol 47: 1-51.

14. Spengler D, Waeber C, Pantaloni C, Holsboer F, Bockaert J, et al. (1993) Differential signal transduction by five splice variants of the PACAP receptor. Nature 365: 170-175.

15. Kenakin T (1995) Agonist-receptor efficacy. II. Agonist trafficking of receptor signals. Trends Pharmacol Sci 16: 232-238.

16. Gether U, Lin S, Kobilka BK (1995) Fluorescent labeling of purified beta 2 adrenergic receptor. Evidence for ligand-specific conformational changes. $J$ Biol Chem 270: 28268-28275.

17. Ghanouni P, Gryczynski Z, Steenhuis JJ, Lee TW, Farrens DL, et al. (2001) Functionally different agonists induce distinct conformations in the $\mathrm{G}$ protein coupling domain of the beta 2 adrenergic receptor. J Biol Chem 276: 24433-24436.

18. Reale V, Hannan F, Hall LM, Evans PD (1997) Agonistspecific coupling of a cloned Drosophila melanogaster D1-like dopamine receptor to multiple second messenger pathways by synthetic agonists. J Neurosci 17 : 6545-6553.

19. Berg KA, Maayani S, Goldfarb J, Scaramellini C, Leff P, et al. (1998) Effector pathway-dependent relative efficacy at serotonin type $2 \mathrm{~A}$ and $2 \mathrm{C}$ receptors: evidence for agonist-directed trafficking of receptor stimulus. Mol Pharmacol 54: 94-104.

20. Swarthout JT, Doggett TA, Lemker JL, Partridge NC (2001) Stimulation of extracellular signal-regulated kinases and proliferation in rat osteoblastic cells by parathyroid hormone is protein kinase C-dependent. $J$ Biol Chem 276: 7586-7592.

21. Palanche T, Ilien B, Zoffmann S, Reck MP, Bucher B, et al. (2001) The neurokinin A receptor activates calcium and cAMP responses through distinct conformational states. J Biol Chem 276: 34853-34861.

22. Azzi M, Charest PG, Angers S, Rousseau G, Kohout $\mathrm{T}$, et al. (2003) Beta-arrestin-mediated activation of MAPK by inverse agonists reveals distinct active conformations for G protein-coupled receptors. Proc Natl Acad Sci U S A 100: 11406-11411.

23. Makita N, Sato J, Manaka K, Shoji Y, Oishi A, et al. (2007) An acquired hypocalciuric hypercalcemia autoantibody induces allosteric transition among active human Ca-sensing receptor conformations. Proc Natl Acad Sci U S A 104: 5443-5448.

24. Jorgensen R, Kubale V, Vrecl M, Schwartz TW, Elling CE (2007) Oxyntomodulin differentially affects glucagon-like peptide-1 receptor beta-arrestin recruitment and signaling through Galpha(s). J Pharmacol Exp Ther 322: 148-154.
25. Urban JD, Vargas GA, von Zastrow M, Mailman RB (2007) Aripiprazole has functionally selective actions at dopamine D2 receptor-mediated signaling pathways. Neuropsychopharmacology 32: 67-77.

26. Wisler JW, DeWire SM, Whalen EJ, Violin JD, Drake MT, et al. (2007) A unique mechanism of beta-blocker action: carvedilol stimulates beta-arrestin signaling. Proc Natl Acad Sci US A 104: 16657-16662.

27. Maillet EL, Pellegrini N, Valant C, Bucher B, Hibert M, et al. (2007) A novel, conformation-specific allosteric inhibitor of the tachykinin NK2 receptor (NK2R) with functionally selective properties. FASEB J 21: 21242134.

28. Pin JP, Kniazeff J, Goudet C, Bessis AS, Liu J, et al. (2004) The activation mechanism of class-C G-protein coupled receptors. Biol Cell 96: 335-342.

29. Davey AE, Leach K, Valant C, Conigrave AD, Sexton PM, et al. (2012) Positive and negative allosteric modulators promote biased signaling at the calcium-sensing receptor. Endocrinology 153: 1232-1241.

30. Brown EM, Vassilev PM, Quinn S, Hebert SC (1999) G-protein-coupled, extracellular $\mathrm{Ca}(2+)$-sensing receptor: a versatile regulator of diverse cellular functions. Vitam Horm 55: 1-71.

31. Makita N, Iiri T (2008) Calcium-sensing receptor. AfCS Nature Molecule Pages: doi:10.1038/ mp.a004000.004001.

32. Pallais JC, Kifor O, Chen YB, Slovik D, Brown EM (2004) Acquired hypocalciuric hypercalcemia due to autoantibodies against the calcium-sensing receptor. $N$ Engl J Med 351: 362-369.

33. Servant G, Tachdjian C, Li X, Karanewsky DS (2011) The sweet taste of true synergy: positive allosteric modulation of the human sweet taste receptor. Trends Pharmacol Sci 32: 631-636.

34. Neubig RR (2007) Missing links: mechanisms of protean agonism. Mol Pharmacol 71: 1200-1202.

35. Kenakin T (1995) Pharmacological proteus? Trends Pharmacol Sci 16: 256-258.

36. Arrang JM, Morisset S, Gbahou F (2007) Constitutive activity of the histamine $\mathrm{H} 3$ receptor. Trends Pharmacol Sci 28: 350-357.

37. Takahashi K, Makita N, Manaka K, Hisano M, Akioka $\mathrm{Y}$, et al. (2012) V2 vasopressin receptor (V2R) mutations in partial nephrogenic diabetes insipidus highlight protean agonism of V2R antagonists. $J$ Biol Chem 287: 2099-2106.

38. Pallais JC, Kemp EH, Bergwitz C, Kantham L, Slovik DM, et al. (2011) Autoimmune hypocalciuric hypercalcemia unresponsive to glucocorticoid therapy in a patient with blocking autoantibodies against the calcium-sensing receptor. J Clin Endocrinol Metab 96: 672680 . 